\title{
Bewegungsverhalten, Kosten mangelnder körperlicher Aktivität und Bewegungsförderung in Österreich
}

\section{Exercise Behavior, Costs of Physical Inactivity, and Physical Activity Promotion in Austria}

\section{(1) $\Theta \Theta$}

\author{
Autoren \\ Susanne Mayer ${ }^{1}$, Rosemarie Felder-Puig² ${ }^{2}$ Erwin Gollner ${ }^{3}$, Thomas Ernst Dorner ${ }^{4,5}$
}

Institute

1 Abteilung für Gesundheitsökonomie, Zentrum für Public Health, Medizinische Universität Wien, Wien, Austria

2 Fachbereich Kinder- und Jugendgesundheit, Institut für Gesundheitsförderung und Prävention (IfGP), Wien, Austria

3 Abteilung Gesundheit, Fachhochschule Burgenland, Pinkafeld, Austria

4 Abteilung für Sozial- und Präventivmedizin, Zentrum für Public Health, Medizinische Universität Wien, Wien, Austria

5 Sozialversicherung öffentlich Bediensteter, Eisenbahnen und Bergbau, Wien, Austria

\section{Schlüsselwörter}

körperliche Inaktivität, gesellschaftliche Kosten, Lebensqualität, Bewegungsförderungsmaßnahmen, Österreich

\section{Key words}

physical inactivity, economic burden, quality of life, physical activity promotion, Austria

\section{Bibliografie}

Gesundheitswesen 2020; 82 (Suppl. 3): S196-S206

DOI 10.1055/a-1219-7701

ISSN $0949-7013$

(C) 2020. The Author(s).

This is an open access article published by Thieme under the terms of the Creative Commons Attribution-NonDerivative-NonCommercial-License, permitting copying and reproduction so long as the original work is given appropriate credit. Contents may not be used for commercial purpose, or adapted, remixed, transformed or built upon. (https://creativecommons. org/licenses/by-nc-nd/4.0/)

\section{Korrespondenzadresse}

Prof. Thomas Ernst Dorner

Abteilung für Sozial- und Präventivmedizin,

Zentrum für Public Health

Medizinische Universität Wien

Kinderspitalgasse 15/I

1090 Wien, Austria

thomas.dorner@meduniwien.ac.at

\section{ZUSAMMENFASSUNG}

Dem Dreischritt „Status quo - Konsequenzen - Maßnahmen“ folgend werden Daten zum Ausmaß körperlicher Aktivität und deren Einflussfaktoren, mögliche gesellschaftliche Kosten mangelnder körperlicher Aktivität sowie Einflussebenen und Maßnahmen zur Bewegungsförderung in Österreich präsentiert. In Österreich ist der Anteil an Personen, die die Bewegungsempfehlungen nicht erfüllen, hoch und es besteht diesbezüglich klar Verbesserungspotenzial, insbesondere in Bezug auf muskelkräftigende Aktivitäten bei Erwachsenen. Je nach Datenquelle erfüllen von den Erwachsenen 42-50\% die Empfehlungen für ausdauerorientierte Bewegung und 18-33\% die für muskelkräftigende Aktivitäten. Gleichzeitig ist die österreichische Bevölkerung im internationalen Vergleich aktiver als jene vieler anderer Länder. Große Unterschiede bestehen im Bewegungsverhalten hinsichtlich demografischer, sozioökonomischer und geografischer Determinanten. Die gesellschaftlichen Folgen durch mangelnde körperliche Aktivität sind beträchtlich, sowohl hinsichtlich verlorener Lebensjahre durch vorzeitigen Tod, als auch den Kosten, die der Allgemeinheit entstehen, beispielsweise an Gesundheitskosten und Produktivitätsverlusten. Die für das Jahr 2017 hochgerechnete Summe von 248 Mio. Euro stellt aufgrund methodischer Schwierigkeiten wahrscheinlich eine Unterschätzung dar. Wissenschaftlich abgesicherte Einflussebenen auf das Bewegungsverhalten umfassen im Sinne der lebens(um)weltortientierten Herangehensweise die Lebenswelten Kindergarten und Schule, Betriebe, Gemeinde/Städte, Pflegewohnheime und die Natur.

\section{ABSTRACT}

In this article, we present the Austrian data on physical activity, the societal costs and outcomes of physical inactivity, and the areas of influence to promote physical activity. Based on an analysis of the status quo and its consequences, suggestions on relevant measures to counter physical inactivity are made. While the proportion of Austrians that do not fulfill the current recommendations on physical activity is high, the Austrian population is physically more active than many populations of other countries. In Austria, major differences in physical activity are associated with demographic, socio-economic, and geographic factors. The eco- 
nomic burden related to physical inactivity is considerable, with regard to both societal costs and outcomes. This includes major health and social care costs, productivity losses (estimated at around 248 million Euro in 2017 in Austria), and life years lost due to premature death. Evidence-based areas of influence to increase physical activity include kindergartens and schools, the workplace, communities, cities, long-term care facilities, and nature.

\section{Einleitung}

Im Sinne des Dreischritts „Status quo - Konsequenzen - Maßnahmen “ werden im Folgenden Daten zum Ausmaß körperlicher Aktivität und deren Einflussfaktoren, mögliche gesellschaftliche Auswirkungen und Kosten mangelnder körperlicher Aktivität sowie Einflussebenen und Maßnahmen zur Bewegungsförderung in Österreich erläutert und zusammengefasst.

\section{Bewegungsverhalten in Österreich}

Für Österreich stehen Daten über das Ausmaß körperlicher Aktivität aus Befragungen mit repräsentativen Stichproben zur Verfügung. Da diese Daten selbstberichtet sind, unterliegen sie den damit verbundenen Einschränkungen. Zudem sind die Fragen zum Bewegungsverhalten in den verschiedenen Surveys unterschiedlich formuliert und unterschiedliche Methoden für die BefragtenAuswahl wurden angewendet, sodass die Ergebnisse der verschiedenen Untersuchungen nur schwer miteinander vergleichbar sind. Bisher wurde in Österreich das Bewegungsverhalten bei Erwachsenen noch nie 2-mal hintereinander mit den gleichen Fragen erhoben, womit Veränderungen nur bedingt darstellbar sind. Da im Jahr 2017 mit einem Bewegungsmonitoring begonnen wurde, besteht aber die Chance, in Zukunft Bewegungstrends für Österreich darstellen zu können.

\section{Bewegungsverhalten von 11- bis 17-jährigen Schülerinnen und Schülern sowie 15- bis 21-jährigen Lehrlingen}

Die „Health Behaviour in School-aged Children (HBSC)“-Befragung, eine Initiative der Weltgesundheitsorganisation (WHO), wird in Österreich bei 11-, 13-, 15- und 17-Jährigen alle 4 Jahre durchgeführt. Die zuletzt publizierten österreichischen Ergebnisse stammen aus dem Schuljahr 2017/18 [1]. Eine ähnliche Befragung österreichischer Lehrlinge wurde im Schuljahr 2018/19 durchgeführt [2]. Die Empfehlung von täglicher körperlicher Bewegung mit zumindest mittlerer Intensität für jeweils mindestens 1 Stunde [3] wurde jedoch nur bei Schülerinnen und Schülern abgefragt.

Sowohl Schülerinnen und Schüler als auch Lehrlinge wurden danach gefragt, „wie häufig sie normalerweise in ihrer Freizeit körperlich so aktiv sind, dass sie dabei außer Atem kommen und/oder schwitzen“. Zusätzlich wurde erhoben, ob sie Team- oder Einzelsport in einem Sportverein oder ähnlichen Einrichtung betreiben. Als Empfehlung wurde formuliert, dass die jungen Menschen mindestens 4-mal wöchentlich sportlich aktiv sein und/oder in einem Verein Sport betreiben sollen. Die aktuellen Ergebnisse sind in - Tab. 1 dargestellt. Daraus ist ersichtlich, dass jüngere Schülerinnen und Schüler die Bewegungsempfehlungen eher erfüllen als ältere. Lehrlinge betreiben in ihrer Freizeit weniger Sport als ungefähr gleichaltrige Schülerinnen und Schüler bzw. sind seltener in einem Sport- verein aktiv. Durchgängig fallen in den drei in \ Tab. 1 dargestellten Gruppen die relativ großen Geschlechterunterschiede zu Ungunsten der Mädchen auf.

Für die Schülerinnen und Schüler liegen auch Trenddaten vor: So ist zwischen 2010 und 2018 der Anteil an Schülerinnen und Schülern, die an mindestens 4 Tagen pro Woche für jeweils mindestens 1 Stunde körperlich aktiv sind, in allen Altersgruppen gestiegen, bei Mädchen deutlicher als bei Burschen > Tab. 2, [1].

\section{Bewegungsverhalten von Erwachsenen und älteren Erwachsenen}

Bei Personen ab 15 Jahren wurde das Bewegungsverhalten im Rahmen der Gesundheitsbefragung 2014 erhoben, inkludiert waren in diese 15770 Personen [4]. Dafür wurde der EHIS-PAQ (Physical Activity Questionnaire of the European Health Interview Survey) eingesetzt. Dies ist ein 8-Item Fragebogen, mit dem nach der körperlichen Aktivität während der Arbeit, nach dem aktiven Mobilitätsverhalten, nach ausdauerbetonten körperlichen Aktivitäten in der Freizeit und nach muskelkräftigenden Aktivitäten gefragt wird. Es wurden nur Aktivitäten gezählt, die länger als 10 Min. am Stück dauerten [5] Gemäß dieser Untersuchung waren in der österreichischen Allgemeinbevölkerung im Alter ab 15 Jahren während der Arbeit knapp die Hälfte (47,7\%) meistens und 40,1 \% kaum körperlich aktiv; 12,2\% gaben an, keiner Arbeit nachzugehen. Dazu ist anzumerken, dass Bewegung während der Arbeit nicht unbedingt mit Gesundheitseffekten verbunden ist.

Die Hälfte aller Befragten (50,1\%) erfüllte die Minimalempfehlungen für Ausdaueraktivitäten in der Freizeit von zumindest 150 Min. Bewegung mit mindestens mittlerer Intensität pro Woche und ein Drittel (32,1\%) erfüllte die minimalen Empfehlungen für muskelkräftigende Aktivitäten von zumindest 2-mal pro Woche. 74,1 \% der österreichischen Allgemeinbevölkerung waren entweder in der Arbeit meistens körperlich aktiv und/oder erfüllten die wöchentlich zumindest empfohlenen $150 \mathrm{Min}$. an ausdauerbetonter Bewegung mit mittlerer Intensität während der Freizeit [6, 7].

Beim österreichischen Bewegungsmonitoring 2017 wurden 4000 Personen ab 15 Jahren zum Bewegungsverhalten gefragt. Dabei wurde der GPAQ (Global Physical Activity Questionnaire) eingesetzt. Hier wurden ebenfalls nur Aktivitäten inkludiert, die mindestens 10 Min. am Stück dauerten. Gemäß dieser Untersuchung erfüllten 42 \% der Österreicherinnen und Österreicher die Mindestempfehlungen von zumindest $150 \mathrm{Min}$. ausdauerbetonter Bewegung mit mittlerer oder 75 Min. ausdauerbetonter Bewegung mit höherer Intensität pro Woche in ihrer Freizeit. Dies war bei $46 \%$ der Männer und 38\% der Frauen der Fall [8]. Muskelkräftigende Aktivitäten mindestens einmal wöchentlich wurden von $18 \%$ der Bevölkerung (23\% der Männer und 14\% der Frauen) angegeben [9].

Im Rahmen des Eurobarometers 472 der Europäischen Kommission wurde das Bewegungsverhalten der EU-Bevölkerung erhoben 
- Tab. 1 Anteil der älteren Kinder und Jugendlichen in Österreich, die verschiedene Aspekte der Bewegungsempfehlungen erfüllen

\begin{tabular}{|c|c|c|c|c|c|c|}
\hline Erhebung & Jahr & $\begin{array}{l}\text { Stichprobe/ } \\
\text { Population }\end{array}$ & Bewegungsempfehlung & Burschen & Mädchen & gesamt \\
\hline \multirow[t]{3}{*}{ HBSC } & $2017 / 18$ & \multirow{3}{*}{$\begin{array}{l}\text { 4196 Schülerinnen und } \\
\text { Schüler, } 11 \text { und } 13 \\
\text { Jahre alt }\end{array}$} & Tägliche körperliche Bewegung * & $27,2 \%$ & $17,1 \%$ & $22,2 \%$ \\
\hline & & & $\begin{array}{l}4 \text { Mal pro Woche oder öfter } \\
\text { sportlich aktiv** }\end{array}$ & $70,9 \%$ & $63,9 \%$ & $67,4 \%$ \\
\hline & & & $\begin{array}{l}\text { Teilnahme an organisierten } \\
\text { Team- oder Einzelsportaktivitä- } \\
\text { ten, } 2 \text { Mal pro Woche oder öfter }\end{array}$ & $63,5 \%$ & $47,8 \%$ & $55,7 \%$ \\
\hline \multirow[t]{3}{*}{ HBSC } & $2017 / 18$ & \multirow{3}{*}{$\begin{array}{l}3293 \text { Schülerinnen und } \\
\text { Schüler, } 15 \text { und } 17 \\
\text { Jahre alt }\end{array}$} & Tägliche körperliche Bewegung * & $14,0 \%$ & $6,3 \%$ & $9,2 \%$ \\
\hline & & & $\begin{array}{l}4 \text { Mal pro Woche oder öfter } \\
\text { sportlich aktiv** }\end{array}$ & $53,7 \%$ & $35,2 \%$ & $42,1 \%$ \\
\hline & & & $\begin{array}{l}\text { Teilnahme an organisierten } \\
\text { Team- oder Einzelsportaktivitä- } \\
\text { ten, } 2 \text { Mal pro Woche oder öfter }\end{array}$ & $45,9 \%$ & $24,4 \%$ & $32,5 \%$ \\
\hline \multirow[t]{2}{*}{ Lehrlingsstudie } & $2018 / 19$ & \multirow[t]{2}{*}{$\begin{array}{l}2001 \text { Lehrlinge, 15-21 } \\
\text { Jahre alt }\end{array}$} & $\begin{array}{l}4 \text { Mal pro Woche oder öfter } \\
\text { sportlich aktiv** }\end{array}$ & $32,4 \%$ & $16,1 \%$ & $24,8 \%$ \\
\hline & & & $\begin{array}{l}\text { Teilnahme an organisierten } \\
\text { Team- oder Einzelsportaktivitä- } \\
\text { ten, } 2 \text { Mal pro Woche oder öfter }\end{array}$ & $28,8 \%$ & $16,8 \%$ & $23,2 \%$ \\
\hline
\end{tabular}

${ }^{*}$ mit zumindest mittlerer Intensität, jeweils mindestens 1 Stunde; ${ }^{*}$ in der Freizeit, körperliche Aktivität mit Schwitzen und/oder außer Atem kommen. HBSC = Health Behaviour in School-aged Children. Quellen: Eigene Darstellung nach [1, 2].

[7]. In Österreich wurden dabei im Jahr 2017 insgesamt 1021 Personen ab 15 Jahren befragt. Es wurde ein Fragebogen eingesetzt, der zwar an den IPAQ (International Physical Activity Questionnaire) angelehnt, aber deutlich modifiziert worden war. Der Anteil der Bevölkerung, der die Bewegungsempfehlungen erfüllte, wurde nicht berechnet. Entsprechend dem Eurobarometer gaben 48 \% der Österreicherinnen und Österreicher an, sich regelmäßig oder ziemlich regelmäßig zu bewegen oder Sport zu betreiben. $42 \%$ berichteten, „regelmäßig“ oder „ziemlich regelmäßig anderwärtig körperlich aktiv“ zu sein, womit Tätigkeiten wie Radfahren, Tanzen, Gartenarbeiten etc. gemeint waren. Der Anteil der körperlich Aktiven ist gemäß Eurobarometer in Österreich seit der Vorerhebung im Jahr 2013 deutlich gesunken. Weiters berichteten 13\% der Befragten in Österreich, dass sie sich wöchentlich an 4-7 Tagen mit höherer Intensität (Tragen schwerer Gegenstände, Graben, Aerobic oder schnelles Radfahren) bewegten und weitere $36 \%$ taten dies an 1-3 Tagen. Bewegung mit mittlerer Intensität (Tragen leichterer Gegenstände, Radfahren mit normaler Geschwindigkeit oder Doppel-Tennis spielen) wurde von $22 \%$ der Österreicherinnen und Österreicher an 4-7 Tagen pro Woche angegeben und von weiteren $41 \%$ an 1-3 Tagen [7]. In Tab. 3 sind die Ergebnisse der österreichweiten Bewegungsbefragungen zusammengefasst.

\section{Einflussfaktoren auf das Bewegungsverhalten}

Geschlechtsunterschiede

In allen zitierten österreichischen Datenquellen $[1,6,8,10]$ berichten männliche Jugendliche und Männer häufiger, körperlich aktiv zu sein als weibliche Jugendliche und Frauen.

\section{Alter}

Beim Zusammenhang zwischen Bewegung und Alter zeigt sich ein differenziertes Bild. Bei Kindern und Jugendlichen ist mit steigen- dem Alter eine deutliche Abnahme des Bewegungsumfangs zu verzeichnen [1]. Auch bei Erwachsenen kommt es mit steigendem Alter tendenziell zu einer Abnahme. So erfüllen von den 15- bis unter 20 -Jährigen $57,5 \%$, von den 30 - bis unter 65 -Jährigen $49,0 \%$ und von den mindestens 65-Jährigen 45,3\% die Mindestempfehlungen für Ausdaueraktivitäten. Bei den muskelkräftigenden Aktivitäten sind die 15 - bis unter 30 -Jährigen die Aktivsten (43,7\% dieser Altersgruppe berichten über muskelkräftigende Aktivitäten 2 Mal in der Woche), gefolgt von den über 65-Jährigen (32,9\%) und den 30 - bis unter 65 -Jährigen (29,5\%). Andererseits sind es jedoch die 30- bis unter 65-Jährigen, die über die meiste körperliche Aktivität bei der Arbeit berichten (48,4\% dieser Altersgruppe sind hier meistens körperlich aktiv), gefolgt von den über 65-Jährigen (hier sind es $44,6 \%$ ) und den 15 - bis unter 30 -jährigen (hier sind es $38,8 \%$ ) [6].

\section{Regionale Unterschiede}

In früheren Datenquellen wurde innerhalb von Österreich ein deutliches West-Ost-Gefälle des Bewegungsverhaltens berichtet [3], das einem sehr ähnlichen Muster wie der Epidemiologie von Adipositas, Diabetes mellitus Typ 2 und der Mortalität durch HerzKreislauferkrankungen [11] folgte. Dieses klare Ost-West-Gefälle ist bei jüngeren Datenquellen nicht mehr so eindeutig zu sehen. Gemäß der österreichischen Gesundheitsbefragung (2014) [10] erfüllen Personen aus den Bundesländern Salzburg, Tirol und Vorarlberg (mit jeweils 60,2, 60,0 und 58,8\%) am häufigsten die Empfehlungen für Ausdaueraktivitäten während der Freizeit. Von der Bevölkerung in den Bundesländern Kärnten, Steiermark und Niederösterreich werden die Bewegungsempfehlungen von rund $50 \%$ $(53,4,52,2$ und 48,0\%) erreicht. In den Bundesländern Burgenland, Oberösterreich und Wien sind es weniger als $50 \%$ (46,2, 45,6 und 44,9\%). Bezüglich muskelkräftigender Aktivitäten erfüllen Bewohner/innen der Bundesländer Salzburg (39,0\%) und Steiermark 
(36,3\%) am häufigsten die Bewegungsempfehlungen und Personen der Bundesländer Burgenland (29,7\%) und Oberösterreich (29,4\%) am seltensten (eigene Berechnungen nach [10]).

Auch im österreichischen Gesundheitsmonitoring sind deutliche Unterschiede zwischen den Bundesländern zu erkennen. Gemäß dieser Erhebung erfüllen Personen aus den Bundesländern Burgenland (45,9\%), Kärnten (45,6\%), und Salzburg (44,9\%) am ehesten die Empfehlungen für Ausdauerbewegung während der Freizeit, im Mittelfeld liegen Tirol (44,6\%), Vorarlberg (44,3\%) und Oberösterreich $(40,0 \%)$ und gefolgt von der Steiermark $(39,6 \%)$, Niederösterreich (39,1\%) und Wien (38,4\%) Zweimal wöchentlich muskelkräftigende Aktivitäten machen am häufigsten Personen aus den Bundesländern Wien (18,1\%) und Niederösterreich (16,2\%). Geringer ist der Anteil bei Personen aus Tirol (7,5\%) (eigene Berechnungen nach [9]).

\section{Bildung und Wohlstand}

In der österreichischen Gesundheitsbefragung zeigte sich ein deutlicher Zusammenhang zwischen Bewegungsumfang und Bildungsgrad. So war mit höherem Bildungsgrad der Anteil der Personen, die die Bewegungsempfehlungen erfüllten, höher. Lediglich bei den Männern waren es die Pflichtschulabsolventen, die die Empfehlungen bezüglich muskelkräftigender Aktivitäten beinahe so häufig erfüllten wie Männer mit tertiärer Bildung. Der Bildungsgradient war bei den Frauen stärker ausgeprägt als bei den Männern, - Tab. 4 (eigene Berechnungen nach [10]).

- Tab. 2 Anteil der Schülerinnen und Schüler, die mindestens 4 Tage in der Woche zumindest 1 Stunde körperlich aktiv sind - Entwicklung seit 2010.

\begin{tabular}{|l|l|l|l|}
\hline & $\mathbf{2 0 1 0}$ & $\mathbf{2 0 1 4}$ & $\mathbf{2 0 1 8}$ \\
\hline Mädchen & $28,8 \%$ & $29,5 \%$ & $33,4 \%$ \\
\hline Burschen & $54,3 \%$ & $49,6 \%$ & $55,8 \%$ \\
\hline 11-Jährige & $57,6 \%$ & $52,6 \%$ & $61,5 \%$ \\
\hline 13-Jährige & $46,7 \%$ & $44,7 \%$ & $48,3 \%$ \\
\hline 15-Jährige & $29,4 \%$ & $30,3 \%$ & $35,0 \%$ \\
\hline 17-Jährige & $22,5 \%$ & $22,2 \%$ & $24,0 \%$ \\
\hline \multicolumn{2}{|l}{ Quelle: Eigene Darstellung nach [1]. } \\
\hline
\end{tabular}

Auch im österreichischen Bewegungsmonitoring zeigte sich ein klarer Zusammenhang zwischen Bildung und Bewegung. Es berichteten 39\% der Personen mit Universitäts- oder Fachhochschulabschluss davon, in ihrer Freizeit Bewegung mit höherer Intensität zu machen, bei Personen mit Matura waren es $45 \%$, bei jenen mit Berufsbildender mittlerer Schule (BMS) oder Fachschule 24\%, bei Personen mit Lehre oder Berufsschule $20 \%$ und bei jenen mit Pflichtschule $12 \%$. Darüber hinaus war hier ein klarer Gradient nach Netto-Haushaltseinkommen und Berufsstatus zu sehen. Ebenfalls ein deutlicher Gradient nach Bildungsstatus war bei jenem Anteil an Personen, die mindestens einmal wöchentlich muskelkräftigenden Aktivitäten nachgingen, zu erkennen. Dieser betrug bei Personen mit Universitäts- oder Fachhochschulabschluss 33\%, bei Personen mit Matura 37\%, bei jenen mit BMS oder Fachschule 16\%, bei Personen mit Lehre oder Berufsschule 13\% und bei jenen mit Pflichtschule $10 \%$. Auch hier zeigte sich ein Zusammenhang mit dem Berufsstatus und dem Netto-Haushaltseinkommen [9]. Ähnliche Ergebnisse liegen auch für ältere Kinder und Jugendliche vor: Separate Analysen der österreichischen HBSC-Daten 2018 [1] zeigen, dass die Wahrscheinlichkeit, dass Schülerinnen und Schüler regelmäßig Sport betreiben und/oder in einem Sportverein aktiv sind, mit zunehmendem Wohlstand ihrer Familien steigt.

\section{Bewegungsverhalten im internationalen Vergleich}

Österreichische Kinder im Alter von 11 und 13 Jahren lagen gemäß der WHO-HBSC-Befragung im Jahr 2013/14 ungefähr im Durchschnitt aller 42 (vorwiegend europäischen) Länder, in denen diese Untersuchung durchgeführt wurde [12]: Bei den 11-Jährigen lag Österreich auf Rang 14 und bei den 13-Jährigen auf Rang 18. Bei den 15-Jährigen lag Österreich mit Rang 37 jedoch deutlich unter dem Durchschnitt. Länder mit wesentlich höherem Aktivitätsniveau bei den 11- bis 15-jährigen waren bspw. Bulgarien, Albanien, die Ukraine oder Spanien [12].

Bei den Erwachsenen zeigt ein internationaler Vergleich österreichischer Daten aus dem Eurobarometer 472 [7], dass der Bewegungsumfang in Österreich innerhalb der EU-28 etwas über dem Durchschnitt liegt. In der EU berichteten $46 \%$ davon, nie Sport zu betreiben oder sich zu bewegen, in Österreich waren es $40 \%$. Auch der Anteil der Personen, die nie anderen körperlichen Aktivitäten (zusätzlich zu Sport) wie Radfahren, Tanzen oder Gartenarbeiten

\Tab. 3 Anteil der erwachsenen österreichischen Bevölkerung, der die Bewegungsempfehlungen erfüllt.

\begin{tabular}{|c|c|c|c|c|c|c|}
\hline Erhebung & Jahr & $\begin{array}{l}\text { Stichprobe/ } \\
\text { Population }\end{array}$ & Bewegungsempfehlung & Männer & Frauen & gesamt \\
\hline \multirow[t]{2}{*}{ AT-HIS } & 2014 & \multirow[t]{2}{*}{$\begin{array}{l}15770 \\
\text { Personen ab } \\
15 \text { Jahren }\end{array}$} & $\begin{array}{l}\text { Ausdauerorientierte Bewegung, } \\
\text { zumindest } 150 \text { Min. pro Woche mit } \\
\text { zumindest mittlerer Intensität oder } \\
\text { Radfahren }\end{array}$ & $53,1 \%$ & $47,2 \%$ & $50,1 \%$ \\
\hline & & & $\begin{array}{l}\text { Muskelkräftigende Aktivitäten } \\
\text { mindestens 2-mal pro Woche }\end{array}$ & $36,8 \%$ & $30,0 \%$ & $33,3 \%$ \\
\hline \multirow[t]{2}{*}{ Bewegungsmonitoring } & 2017 & \multirow[t]{2}{*}{$\begin{array}{l}4000 \text { Personen } \\
\text { ab } 15 \text { Jahren }\end{array}$} & $\begin{array}{l}\text { Mindestens } 150 \text { Min. pro Woche } \\
\text { Bewegung mit mittlerer oder } 75 \text { Min. } \\
\text { pro Woche mit höherer Intensität in } \\
\text { der Freizeit oder beim Sport }\end{array}$ & $46 \%$ & $38 \%$ & $42 \%$ \\
\hline & & & $\begin{array}{l}\text { Muskelkräftigende Aktivitäten } \\
\text { mindestens einmal wöchentlich }\end{array}$ & $23 \%$ & $14 \%$ & $18 \%$ \\
\hline
\end{tabular}


\Tab. 4 Anteil der erwachsenen österreichischen Bevölkerung, der die Bewegungsempfehlungen erfült, nach Bildungsstatus.

\begin{tabular}{|c|c|c|c|c|}
\hline Bewegungsempfehlung & Höchste abgeschlossene Schulbildung & Männer & Frauen & gesamt \\
\hline \multirow{3}{*}{$\begin{array}{l}\text { Ausdauerorientierte Bewegung, zumindest } \\
150 \text { Min. pro Woche mit zumindest mittlerer } \\
\text { Intensität oder Radfahren }\end{array}$} & Pflichtschule & $49,5 \%$ & $39,4 \%$ & $43,2 \%$ \\
\hline & Matura oder abgeschlossene Lehre & $49,4 \%$ & $46,8 \%$ & $48,2 \%$ \\
\hline & Universität oder Fachhochschule & $62,1 \%$ & $55,0 \%$ & $58,4 \%$ \\
\hline \multirow{3}{*}{$\begin{array}{l}\text { Muskelkräftigende Übungen mindestens } \\
\text { 2-mal pro Woche }\end{array}$} & Pflichtschule & $39,1 \%$ & $26,2 \%$ & $31,1 \%$ \\
\hline & Matura oder abgeschlossene Lehre & $33,4 \%$ & $28,8 \%$ & $31,3 \%$ \\
\hline & Universität oder Fachhochschule & $41,5 \%$ & $35,2 \%$ & $38,2 \%$ \\
\hline
\end{tabular}

Quelle: Eigene Berechnungen nach [10].

nachgehen, war in Österreich mit $27 \%$ geringer als im EU-Durchschnitt (35\%). Im EU-Vergleich ist der Anteil an Personen, die sich viel bewegen, in den skandinavischen Ländern (Finnland, Schweden und Dänemark) sowie in den Niederlanden, Belgien, Luxemburg und Slowenien besonders hoch. Zu den Ländern, deren Einwohner/innen einen auffallend geringen Bewegungsumfang aufweisen, zählen Malta, Portugal, Italien, Zypern, Rumänien, Spanien, Bulgarien und Griechenland [7]. Somit sind in Europa tendenziell ein Nord-Südund ein West-Ost-Gefälle im Bewegungsumfang zu verzeichnen.

\section{Gesellschaftliche Folgen mangelnder körperlicher Aktivität}

Die gesundheitlichen Auswirkungen mangelnder körperlicher Aktivität sind hinlänglich bekannt. Chronische Krankheiten, eingeschränkte Mobilität und mangelnde Belastbarkeit, die durch einen inaktiven Lebensstil mitverursacht werden, sind nicht nur mit dem vorzeitigen Tod, sondern auch mit Einbußen in der Leistungsfähigkeit und dadurch in der Arbeitsfähigkeit und damit massiven gesellschaftlichen Konsequenzen verbunden [13].

\section{Todesfälle, Lebensqualität, Disbability-Adjusted Life Years}

Ein Konzept, das die Krankheitslast sowohl hinsichtlich „vorzeitig verlorener Lebensjahre“ durch Tod als auch hinsichtlich der „in Krankheit verbrachten Lebensjahren“ misst, ist jenes der „Disability Adjusted Life Years“ (DALYs) [14]. DALYs sind eine Maßzahl für die verlorenen gesunden Lebensjahre [15]. Weltweit werden die Verluste aufgrund mangelnder Bewegung auf rund 13,4 Mio. DALYs, also „verlorene gesunde Lebensjahre“, geschätzt, für die Europäische Union auf 2,1 Mio. und für Österreich auf 20300 DALYs [15]. Innerhalb der Bevölkerung der Europäischen Union entfallen pro 100000 Personen 30 vorzeitige Todesfälle auf nicht-ansteckende Erkrankungen, die Bewegungsmangel zuzuschreiben sind [15]. Von einem vorzeitigen Todesfall wird gesprochen, wenn der Tod vor dem durchschnittlichen Sterbealter der entsprechenden Bevölkerung eintritt. Sowohl die Anzahl der Todesfälle als auch jene der DALYs aufgrund mangelnder Bewegung hat weltweit in den letzten Jahren zugenommen. Innerhalb einer Dekade sind die Todesfälle aufgrund geringer körperlicher Aktivität um 18,9\% gestiegen, die DALYs um 17,4\% [16]. Im Jahr 2015 waren ischämische Herzkrankheiten und ischämische Schlaganfälle die häufigsten Ursachen für entsprechende Todesfälle. DALYs hingegen wurden am öftesten durch ischämische Herzkrankheiten und Diabetes mellitus Typ 2 verursacht.
In der Liste der führenden Risikofaktoren, vorzeitig zu sterben, liegt mangelnde Bewegung auf Platz 4 [17]. Zudem würde der vollständige Wegfall körperlicher Inaktivität internationalen Schätzungen zufolge die Lebenserwartung der Weltbevölkerung um 0,68 Jahre erhöhen [18]. Die in Zusammenhang mit mangelnder Bewegung stehenden Todesfälle haben aber auch Auswirkungen, die Produktivitätsverluste, entgangene Löhne und Gehälter sowie soziale Netzwerke betreffen. Wie viel Lebensqualität durch mangelnde Bewegung eingebüßt wird und welche weiteren sozialen Auswirkungen ein inaktiver Lebensstil für den Einzelnen und die Bevölkerung mit sich bringt, ist jedoch schwierig zu quantifizieren. Während bspw. in verschiedenen Studien gezeigt wird, dass Bewegung im empfohlenen Ausmaß zu höherer Lebensqualität führt, gibt es nur wenige Analysen zum Zusammenhang zwischen mangelnder Bewegung und Lebensqualität. Laut einer in den USA durchgeführten Studie berichten Erwachsene mit mangelnder Bewegung doppelt so häufig von einer schlechten gesundheitsbezogenen Lebensqualität wie Personen, die körperlich aktiv sind [19].

\section{Kosten in unterschiedlichen Sektoren und Produktivitätsverluste}

Mangelnde körperlicher Aktivität hat Auswirkungen auf die Kosten im Gesundheitssystem und andere betroffene Bereiche und Sektoren in der Gesellschaft. Kostendaten sind eine mögliche Informationsbasis für die Gesundheitsplanung. Darüber hinaus können sie auch für verstärkte gesundheitspolitische und sektorenübergreifende Bemühungen zur Steigerung körperlicher Aktivität eingesetzt werden [20].

Aus gesundheitsökonomischer Sicht lassen sich die Auswirkungen von körperlicher Inaktivität in Kosten bemessen, die in den unterschiedlichen Sektoren einer Volkswirtschaft entstehen [21]. Zu den Kosten im Gesundheitssektor zählen erhöhter Ressourcenverbrauch durch Arztbesuche, Krankenhausaufenthalte, Arzneimittel und Diagnostik. Darunter fallen auch die Behandlungskosten von Erkrankungen, die als Folge von mangelnder Bewegung auftreten, wie bspw. Herz-Kreislauferkrankungen, Diabetes mellitus Typ 2, einige Krebserkrankungen, Rückenschmerzen oder Depressionen [22]. Weitere Kosten entstehen v. a. durch Produktivitätsverluste am Arbeitsmarkt. Diese entstehen nicht nur durch Krankenstandstage, Arbeitszeitverkürzungen und Frühpensionierungen (Absentismus) für die Arbeitgeber/innen und Krankenversicherungen, sondern auch durch verminderte Leistungsfähigkeit am Arbeitsplatz (Präsentismus). Außerdem umfassen die Auswirkungen auch Einkommensverluste durch vorzeitigen Tod. Ebenso können Produktivitätsverluste bei pflegenden Angehörigen entstehen, die anstel- 
le der informellen Betreuung einer formalen Beschäftigung nachgehen könnten. Kosten für die Patientinnen/Patienten und ihre Familien fallen darüber hinaus auch durch Zahlungen aus eigener Tasche, z. B. für Selbstbehalte bei Arztbesuchen oder für Medikamente und Transporte, an. Denkbar ist, dass im Zusammenhang mit den gesundheitlichen Konsequenzen von körperlicher Inaktivität aus gesellschaftlicher Perspektive auch Kosten in weiteren Sektoren entstehen. So können bspw. Depressionen, die als Folge von mangelnder Bewegung auftreten, zu vermehrten Polizeikontakten und somit erhöhten Kosten im Justizbereich führen oder erhöhten Förderbedarf bei Kindern und Jugendlichen nötig machen, der höhere Kosten im Bildungsbereich nach sich zieht [23, 24]. Schließlich entstehen auch intangible Kosten. Damit sind Auswirkungen auf die Lebensqualität von Betroffenen und deren Angehörigen gemeint, die durch Schmerzen und Einschränkungen verursacht werden, die jedoch monetär nur schwer zu bemessen sind.

Während es eine wachsende Zahl von Krankheitskostenstudien zu chronischen Erkrankungen gibt, die im Zusammenhang mit körperlicher Inaktivität stehen, wie z. B. zu Adipositas, Diabetes mellitus Typ 2 oder kardiovaskulären Erkrankungen, finden sich nur wenige Studien, die die ökonomischen Konsequenzen von mangelnder Bewegung bei Kindern und Jugendlichen untersuchen. Gleichzeitig scheint die Kindheit eine zu frühe Lebensphase zu sein, um Gesundheitskosteneinsparungen aufgrund präventiver Effekte feststellen zu können [25, 26]. Dennoch muss dem Individuum selbst sowie den gesellschaftlichen Vertreterinnen bzw. Vertretern bewusst sein, dass körperliche Inaktivität einen wichtigen modifizierbaren Risikofaktor für viele chronische Krankheiten darstellt $[26,27]$, die bei ausreichender Bewegung erst später im Lebensverlauf auftreten [25].

\section{Kosten aufgrund mangelnder Bewegung international}

In einer internationalen Studie [20] wurden die Kosten für koronare Herzkrankheiten, Herzinfarkt, Diabetes mellitus Typ 2, Brustkrebs und Dickdarmkrebs, die jenen Krankheiten, die durch Bewegungsmangel entstehen, zuzurechnen sind, für das Jahr 2013 geschätzt. Weltweit belaufen sich diese Gesundheitskosten demnach auf rund 53,8 Mrd. internationale Dollar, von denen circa 9,7 Mrd. für private Haushalte anfallen. Außerdem wurden die Produktivitätsverluste mittels eines sogenannten Friktionskostenansatzes, der einen Ersatz verstorbener Arbeitskräfte auf dem Arbeitsmarkt innerhalb von 3 Monaten annimmt, approximiert und mit weiteren 13,7 Mrd. internationale Dollar beziffert [20].

\section{Kostenschätzung für Österreich}

Eine erste Grundlage zur Schätzung der möglichen Kosten, die der Allgemeinheit aufgrund mangelnder körperlicher Aktivität entstehen, stellt die Studie von Weiß et al. dar [28]; aktualisiert [29]. In den österreichischen Empfehlungen für gesundheitswirksame Bewegung 2010 wurden Schätzungen für Österreich, basierend auf internationalen Modellen, vorgenommen [3].

In der globalen Studie von Ding et al. [20] wurden die Kosten, die Österreich durch mangelnde körperliche Aktivität entstehen, auf $0,47 \%$ (zwischen 0,05 und $1,83 \%$ als Extremszenarien) der Gesundheitsausgaben geschätzt. Auf Basis österreichischer Daten für das Jahr 2017 [30] entspricht dies rund 181 Mio. EUR an Gesund- heitskosten durch Bewegungsmangel (19 Mio. EUR bis 0,7 Mrd. EUR). Überträgt man hingegen eine US-amerikanische, weniger konservative Schätzung, der zufolge 2,4 bis $5 \%$ der Gesundheitsausgaben mangelnder körperlicher Aktivität zuzuschreiben sind [31], auf Österreich, so würden das Gesundheitskosten von 0,9 bis 1,2 Mrd. EUR jährlich bedeuten. Allerdings ist die Übertragbarkeit des US-Ansatzes auf Österreich aufgrund diverser Systemunterschiede kritisch zu sehen. Wird eine britische Berechnungsmethode auf Österreich umgelegt [13], belaufen sich die geschätzten Gesundheitskosten aufgrund von Erkrankungen im Zusammenhang mit mangelnder Aktivität auf rund 0,6 Mrd. EUR. Noch höher sind die Kosten durch körperliche Inaktivität. In Österreich werden die dadurch im Gesundheitswesen anfallenden Kosten (2013) auf 1,31,9 Mrd. Euro geschätzt (das entspricht 3,6-5,5\% der Gesundheitsausgaben) [32].

Auf Basis der Berechnungen von Ding et al. fielen 2017 für Österreich weitere 57 Mio. EUR (5 Mio. EUR bis 198 Mio. EUR) an Kosten durch verlorene Produktivität im Zusammenhang mit vorzeitigen Todesfällen an [20]. Produktivitätsverluste aufgrund von Krankenständen, Präsentismus, Absentismus und informellen Pflegetätigkeiten konnten in diesen Berechnungen nicht berücksichtigt werden, was zu einer Unterschätzung der Produktivitätsverluste führt. Ebenso nicht einbezogen sind Kosten, die z. B. durch die Folgeerscheinungen mangelnder körperlicher Aktivität, wie etwa Übergewicht und Adipositas, verursacht werden. In Österreich waren 2017 die meisten Krankenstandstage (21,4\%) von Frauen und Männern auf Beschwerden des Muskel-Skelett-Systems und des Bindegewebes - hier v. a. auf chronische Rückenschmerzen zurückzuführen [33]. Da es Hinweise gibt, dass sowohl körperliche Inaktivität als auch ein sehr hohes Aktivitätsniveau für Rückenschmerzen verantwortlich sein können [34], könnten hier noch weitere beträchtliche Kosten dazukommen, die in den verschiedenen Analysemodellen jedoch selten berücksichtigt werden. Die berechnete Summe (nach [20]) von 248 Mio. EUR (23 Mio. EUR bis 0,9 Mrd. EUR) stellt somit jedenfalls eine zu niedrige Einschätzung der Kosten dar, die der Gesellschaft in Österreich durch mangelnde Bewegung entstehen.

\section{Bewegungsförderung}

Obwohl der Gesundheitsgewinn durch körperliche Aktivität wissenschaftlich gut dokumentiert ist und auf breite gesellschaftliche Akzeptanz stößt, fällt es vielen Menschen schwer, einen körperlich aktiven Lebensstil zu führen. Dies liegt allerdings nicht nur an der individuellen Entscheidung von Personen, sondern auch an den Verhältnissen, bspw. infrastrukturelle Rahmenbedingungen die Bewegung erlauben [35].

Bewegungsförderung beinhaltet alle Maßnahmen, die gezielt und systematisch zur Verbesserung des Bewegungsverhaltens gesetzt werden, um die Gesundheit einzelner Menschen sowie die Gesundheit der Bevölkerung insgesamt zu stärken [36]. Die WHO fordert daher in ihrem „Globalen Aktionsplan für Bewegung 20182030“ nationale strategische Maßnahmen zur Verbesserung der sozialen, kulturellen, wirtschaftlichen und politischen Rahmenbedingungen für körperliche Aktivität [37].

Auf politischer Ebene wird die Forderung der WHO in Österreich mit den „Gesundheitszielen Österreich“, konkret mit dem achten 


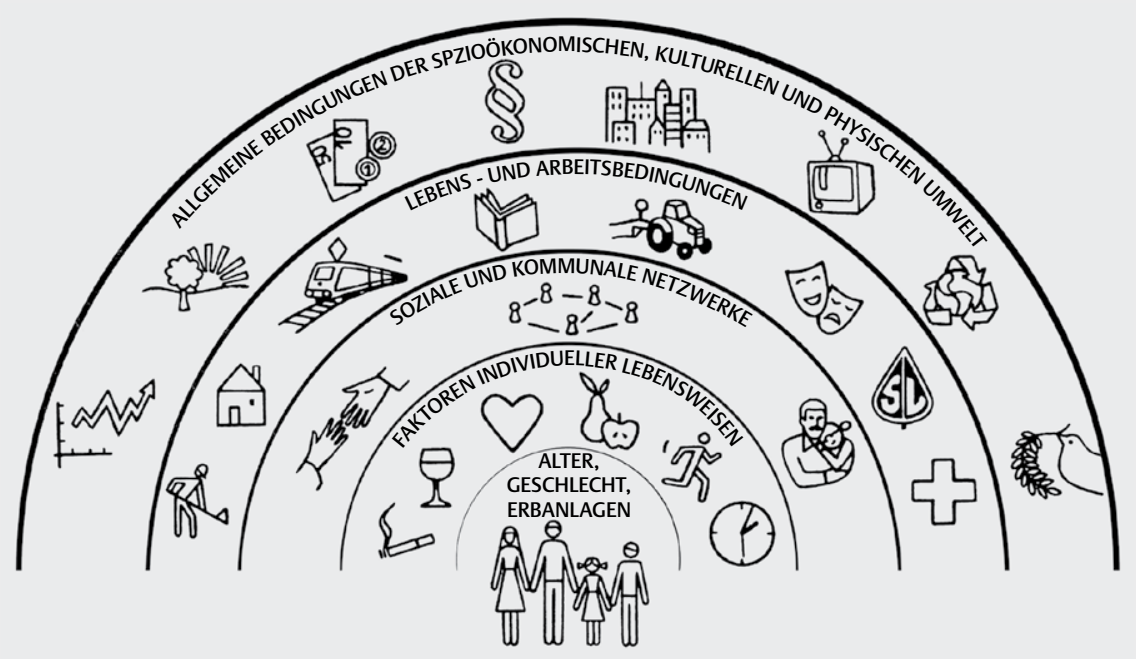

Abb. 1 Einflussebenen auf das Bewegungverhalten. Quelle: [40]; Abbildung: [59].

Gesundheitsziel „Gesunde und sichere Bewegung im Alltag fördern“, umgesetzt. Darin wird festgehalten, dass insbesondere in Kindergärten, Schulen, Senioren- und Pflegeheimen sowie in Vereinen die Freude an Bewegung und das Wissen um ihre gesundheitsförderliche Bedeutung gestärkt werden sollen [38]. Dieser lebensweltenorientierte Zugang zur Bewegungsförderung erfordert die Einbeziehung aller relevanten politischen bzw. gesellschaftlichen Sektoren. Je nachdem, welche Form körperlicher Aktivität (z. B. aktive Mobilität, freizeitbezogene Bewegungsprogramme) und wo (z. B. Schule, Betrieb) Bewegung gezielt gefördert werden soll, ist es notwendig, auch außerhalb des Sport- und Gesundheitssektors Entscheidungsträgerinnen und Entscheidungsträger (z. B. aus Verkehr und Bildung) einzubinden. Dieser intersektorale Zugang wurde bereits bei der Erstellung des Nationalen Aktionsplans Bewegung (NAP.b) betont [39].

In Abb. 1 sind die unterschiedlichen Einflussebenen auf das Bewegungsverhalten dargestellt. Im Zentrum dieses Modells steht der Mensch mit seinen individuellen Voraussetzungen wie Alter, Geschlecht und Erbanlagen, die als nicht direkt veränderbare Einflussfaktoren gelten. Es gibt aber Einflussfaktoren auf das Bewegungsverhalten, die durch geeignete Maßnahmen direkt verändert werden können. Je weiter außen die Halbkreise dieser Darstellung, desto weniger liegen die Einflussmöglichkeiten im Bereich der Einzelindividuen und umso mehr im Bereich der gesamten Gesellschaft. Folgende veränderbare Faktoren können das Bewegungsverhalten beeinflussen [40]:

1. Individuelle Verhaltens- und Lebensweisen Dazu gehören die individuelle Einstellung zum Thema Bewegung, die Motivation für Bewegung, die Freude an Bewegung sowie Konsequenzerwartungen, Durchhaltevermögen, Selbst- und Zeitmanagement und motorische Fertigkeiten bzw. Bewegungskompetenz.

2. Soziales Umfeld und Netzwerke In diesen Bereich fallen die soziale Unterstützung bei der Ausübung eines körperlich aktiven Lebensstils durch Familie, Freunde und Freundinnen sowie Peergroups, das Vorhandensein informeller Bewegungsgrup- pen und von Aktivitäten im organisierten Rahmen sowie die kulturelle und religiöse Bedeutung von Bewegung.

3. Lebens- und Arbeitsbedingungen Damit sind verhaltens- und verhältnisorientierte Bewegungsförderungsmaßnahmen in den Lebenswelten Kindergarten, Schule, Betrieb, Gemeinde/Stadt, Pflegewohnheim, Gesundheitseinrichtung usw. gemeint.

4. Gebaute Umgebung, sozioökonomisches und politisches Umfeld Bei dieser Einflussebene geht es um die Wohnumgebung, die Wirtschaftslage, das Einkommen (z. B. Leistbarkeit von Bewegungsangeboten), angeleitete Bewegungsräume (z.B. Radwege, Wanderwege, Sportstätten) und Strategien/Aktivitäten initiiert von der Politik, den Medien sowie den Sport- und Gesundheitsorganisationen.

Um eine erfolgreiche Verhaltensänderung in Richtung mehr Bewegung innerhalb der Bevölkerung zu erzielen, ist es notwendig, wissenschaftlich abgesicherte Maßnahmen koordiniert auf allen 4 Einflussebenen zu setzen. Im Folgenden wird im Sinne einer lebens(um) weltorientierten Herangehensweise v. a. auf die zwei äußeren Ebenen des Modells eingegangen. Dabei werden beispielhaft Ansätze dargestellt, die sich in der Praxis bewährt haben und für deren Wirksamkeit es wissenschaftliche Belege gibt.

Bei der Umsetzung von Bewegungsförderung sind die wesentlichen Prinzipien der Gesundheitsförderung wie z. B. die Orientierung an der Zielgruppe, die Partizipation der Zielgruppe und sonstiger Stakeholder sowie die (frühzeitige) Vernetzung mit potenziellen Kooperationspartnern und Kooperationspartnerinnen einzuhalten [41]. Neben bewusster Bewegung in der Freizeit zielen viele Bewegungsförderungsmaßnahmen auf die Steigerung der aktiven Mobilität im Alltag ab. Damit ist jede Form der Fortbewegung aus eigener Muskelkraft, um von A nach B zu kommen, gemeint. Dazu gehören v. a. Zufußgehen und Radfahren, aber auch die Nutzung von Scootern, Tretrollern oder Skateboards [42]. Dabei können moderne Technologien (Schrittzähler und Trackingsysteme) den Spieltrieb fördern sowie „Nudging“ als Anreiz eingesetzt wer- 
den. Nudges sind sanfte „Anstupser“, mit denen man bestimmte Verhaltensweisen, in diesem Fall ein gesundes Bewegungsverhalten, hervorrufen möchte.

\section{Lebenswelt Kindergarten und Schule}

Da im Vorschulalter Lebensgewohnheiten entwickelt werden, spielt Bewegungsförderung während dieser Zeit eine große Rolle. Dabei kann z. B. die Tatsache genutzt werden, dass sich Kinder von Natur aus gerne bewegen [43]. In der Praxis erwies sich bislang v. a. die Kombination aus a) Fortbildungsmaßnahmen des Kindergartenpersonals zum Thema Bewegungsförderung, b) der Sensibilisierung von Erziehungsberechtigten für das Thema Bewegung und c) der Veränderung der materiellen Ausstattung des Kindergartens als wirksam $[36,43]$.

Im Kontext Schule ist die Herausforderung, strukturierte (z. B. Unterrichtsfach „Bewegung und Sport“) und unstrukturierte (z. B. aktive Pausengestaltung) Bewegungsangebote sowie Bewegungsangebote während und außerhalb der Schule (z. B. Vernetzung von Sportangebotsformen in der Schule mit Nachmittagsangeboten in der Ganztagsschule oder in Sportvereinen) miteinander zu kombinieren und aufeinander abzustimmen. Zur Förderung der aktiven Mobilität und des aktiven Spiels ist die Schaffung einer bewegungsfreundlichen Schulumgebung eine Voraussetzung, kombiniert mit einer kontinuierlichen Kompetenzentwicklung des Lehrpersonals zu verschiedenen Aspekten der Bewegungsförderung [36, 44]. Bei Jugendlichen erwies sich bislang der sogenannte „Peergroup-Ansatz“, bei dem junge Menschen zu „Mentoren“ bzw. „Mentorinnen“ ausgebildet werden und andere Jugendliche zu mehr Bewegung motivieren, als effektiv [43].

In den Lebenswelten „Kindergarten“ und „Schule“ gibt es in Österreich bereits zahlreiche Initiativen zur Förderung eines bewegungsaktiven und gleichzeitig umweltfreundlichen Transports von Kindern und Jugendlichen. Maßnahmen, die unter dem Titel „Schulisches Mobilitätsmanagement“ geführt werden, reichen von bewusstseinsbildenden Maßnahmen (z. B. aktiv Kilometer sammeln), über Wettbewerbe und spielerische Aktivitäten (z. B. Schritte zählen, Sammeln von Punkten für zurückgelegte Wege) bis hin zu Vorschlägen zur Verbesserung der Rahmenbedingungen im Schulumfeld (z. B. Rollerabstellplätze) [45].

\section{Lebenswelt Betrieb}

Da Arbeitnehmer/innen durchschnittlich $60 \%$ ihrer Tageszeit am Arbeitsplatz verbringen, kommt dieser Lebenswelt im Hinblick auf Bewegungsförderung große Bedeutung zu [46]. Ähnlich wie in den anderen Lebenswelten ist auch hier eine Kombination aus verschiedenen Aktivitäten besonders wirksam. Dazu gehören a) (Kurs-)Angebote, die alle Beschäftigten ansprechen und zu keiner Ausgrenzung führen (z. B. Nordic Walking, Wandern), b) die Umgestaltung betrieblicher Abläufe (z. B. alternative Pausengestaltung wie Bewegungs- oder Entspannungspausen), c) die Schaffung bewegungsförderlicher Infrastrukturen (z. B. innerbetriebliche Fußwege, Grünflächen mit Motorikgeräten) sowie d) die Förderung von aktiver Mobilität oder die Etablierung von „Stiegen statt Aufzug“ oder „Walk to Talk“ [36, 46, 47].

Eine wesentliche Voraussetzung für das Gelingen von Bewegungsförderungsaktivitäten in der Lebenswelt „Betrieb“ ist das bei- derseitige Engagement der Mitarbeiter/innen sowie der Führungskräfte [46].

Initiativen, die das Thema der aktiven Mobilität betreffen, sind im Setting Betrieb z. B. Zertifizierungsmaßnahmen („Fahrradfreundlicher Betrieb“), die Einführung eines Dienstfahrrads ergänzend zum Dienstauto sowie diverse bewusstseinsstärkende Mobilitätsaktionen („Österreich radelt/walkt zur Arbeit“) [48].

\section{Lebenswelt Gemeinde/Stadt}

Eine Besonderheit und gleichzeitig auch Schwierigkeit dieser Lebenswelt liegt darin, alle Personengruppen, unabhängig von Alter, Geschlecht und sozioökonomischem Status zu erreichen. Wirksame Interventionsbereiche in dieser Lebenswelt sind a) die aktive, sichere Mobilität (z. B. Schaffung verkehrssicherer Rad- und Fußwege), b) die öffentliche Gebäudeplanung (z. B. Stiegenhausgestaltung, Begegnungszonen), c) die Stadtplanung und -gestaltung (z. B. Gestaltung von Spielplätzen, Grünzonen und Bewegungsparks) sowie d) die natürliche Umwelt (z. B. Zugang zu „freien“ Bewegungsflächen) [49].

Im Setting „Gemeinde/Stadt“ ist ein primäres Argument für die Entwicklung von Maßnahmen zur Förderung aktiver Mobilitätsformen die Verbesserung der Lebensqualität durch die Reduktion von Stau, Lärm, Emissionen und Unfällen. Aber auch die Gesundheit durch Bewegung oder die Förderung von sozialen Kontakten sind wesentliche Argumente für die Implementierung von konkreten kommunalen Maßnahmen und Aktionen zur Förderung aktiver Mobilität [42].

\section{Lebenswelt Pflegewohnheim und alternative Wohnform für ältere Menschen}

Ein gesunder und aktiver Lebensstil kann das Altern verzögern und die Anzahl an gesunden Lebensjahren erhöhen. Dabei ist besonders zu berücksichtigen, dass der individuelle gesundheitliche Zustand älterer Menschen so unterschiedlich ist wie ihre individuelle Biografie. In stationären Einrichtungen der Pflege ist es notwendig, die Bedürfnisse von älteren Menschen sowie deren Mobilitätseinschränkungen bei bewegungsförderlichen Maßnahmen zu berücksichtigen, besonders im Hinblick auf die Sturzsicherheit und den Zugang zu barrierefreien Bewegungsflächen [50, 51].

Erfolgreiche angeleitete Interventionsbereiche in dieser Lebenswelt sind a) die Installierung und attraktive Positionierung von Bewegungsboxen (mit Bewegungsmaterialien), b) die Gestaltung architekturgestützter Bewegungs- und Begegnungsräume (z. B. Anlage von einladend gestalteten Rundgängen), c) die Schaffung sturzgesicherter Koordinationsparcours mit vielen Gleichgewichtsübungen, d) begleitete intergenerative Aktivitäten mit Angehörigen oder Kindern sowie e) der Zugang zu barrierefreien Bewegungsflächen [50,52].

Die Integration des Pflegepersonals sowie der Angehörigen ist eine wesentliche Voraussetzung dafür, dass die Bewohner/innen die Bewegungsmöglichkeiten auch tatsächlich nützen.

\section{Lebenswelt Natur}

Aufgrund eines bewegungsarmen Lebensstils und der Zunahme verbauter Flächen entwickelt sich ein zunehmendes Bedürfnis nach einem körperlichen und psychischen Ausgleich durch Erholungsmaßnahmen in Naturgebieten [53]. 
Die Natur als Erlebnisraum bietet allen Altersgruppen die Möglichkeit für ein breites Spektrum an körperlichen Bewegungsformen, am Berg, in flachen Gebieten, in Gewässern etc. und das zu allen Jahreszeiten. Dieses breite Spektrum an Bewegungsmöglichkeiten in der Natur umfasst viele Varianten des Wanderns, des Bergsports sowie eine Reihe von Wassersportarten.

Naturlandschaften können auf vielfältige Art und Weise eine gesundheitsfördernde Wirkung auf den Menschen ausüben. Die Natur unterstützt die körperliche und psychische Gesundheit insbesondere dadurch, dass sie Anreize für Bewegung schafft. Bewegung in der Natur verbessert das psychische Wohlbefinden. So wirkt Bewegung in Naturlandschaften stimmungsaufhellend, senkt die mentale Erschöpfung und verbessert die Erholungsfähigkeit [54].

In Städten stellen schnell erreichbare Grünanlagen wie Parks eine sinnvolle Alternative zu den ländlichen Naturräumen dar.

Interventionsmaßnahmen in der Lebenswelt „Natur“ sind a) die Sicherung von Bewegungsinfrastrukturen mit öffentlichem Zugang, b) die Instandhaltung und Beschilderung der Bewegungsräume in der Natur, c) das Setzen klarer gesetzlicher Regelungen für die Benutzung der Naturräume sowie d) das Angebot einer Ausbildung von Multiplikator/innen zur Animation und sicheren Benützung der Naturräume [55].

\section{Fazit}

In Österreich ist der Anteil an Personen, die die Bewegungsempfehlungen nicht erfüllen, hoch und es besteht diesbezüglich klar Verbesserungspozenzial, insbesondere in Bezug auf muskelkräftigende Aktivitäten bei Erwachsenen. Als positiv ist hervorzuheben, dass es zumindest bei älteren Kindern und Jugendlichen einen positiven Trend in Richtung mehr Bewegung gibt. Außerdem ist die österreichische Bevölkerung im europäischen und internationalen Vergleich aktiver als jene viele anderer Länder. Es gibt in Österreich jedoch große Unterschiede im Bewegungsumfang, das Geschlecht, das Alter, die sozialen Schichten und die geografischen Regionen betreffend. Gleichzeitig fällt beim Vergleich der Ergebnisse für ältere Kinder und Jugendliche sowie Erwachsene auf, dass die Unterschiede zwischen den Geschlechtern im Erwachsenenalter nicht mehr so groß sind wie in der Jugend. Dies hat möglicherweise damit zu tun, dass viele Mädchen in der Pubertät vorübergehend ein negatives Körperselbstbild entwickeln, ihren Körper lieber verstecken wollen und sportliche Aktivitäten deshalb vermeiden.

Die Kosten durch mangelnde körperliche Aktivität aus gesundheitsökonomischer Perspektive sind beträchtlich. Das kostentreibende Element ist dabei die Vielzahl an Erkrankungen, die durch Bewegungsmangel mitverursacht werden. Diese Erkrankungen treten in der Bevölkerung teils sehr häufig auf und es handelt sich um chronische Krankheiten, die naturgemäß einen lang dauernden Verlauf nehmen, was zu direkten Behandlungskosten führt. Zudem fallen aber auch indirekt Kosten aufgrund beeinträchtigter Arbeitsfähigkeit an. Dadurch entstehen Kosten für die betroffenen Personen und deren Angehörige, die Arbeitgeber/innen, die Sozialversicherungen (Kranken- und Pensionskassen) und andere volkwirtschaftliche Sektoren. Abgesehen von den bezifferbaren Kosten ist mangelnde körperliche Aktivität unter anderem auch die Ursache für Beeinträchtigungen der Lebensqualität sowie für - durch sol- che Beeinträchtigung und vorzeitigen Tod - verlorene Lebensjahre. Die für Österreich im Jahr 2017 hochgerechnete Summe von 248 Mio. Euro an gesellschaftlichen Kosten im Zusammenhang mit mangelnder körperlicher Aktivität stellt aufgrund methodischer Schwierigkeiten wahrscheinlich eine Unterschätzung dar.

Bewegungsförderungsmaßnahmen sind dann erfolgreich, wenn es dadurch für Menschen einfach und attraktiv wird, körperlich aktiv zu sein. Das Ziel muss es daher sein, die jeweiligen Lebenswelten der Menschen - dort wo sie wohnen, arbeiten/lernen oder die Freizeit verbringen - so zu verändern, dass es leicht wird, Bewegung in den Alltag zu integrieren. Durch die Zusammenarbeit unterschiedlicher Sektoren (z. B. Sport, Bildung, Verkehr) steigt das Potenzial, positive Veränderungen einzuleiten. Wirkungsvolle Bewegungsförderungsprogramme zeichnen sich darüber hinaus dadurch aus, dass sie auf Verhaltensänderungsmodellen aufbauen und Einflussfaktoren auf das Bewegungsverhalten berücksichtigen. Dazu gehören einerseits die individuelle Lebensweise und anderseits auch das soziale Umfeld, Netzwerke, Lebens- und Arbeitsbedingungen und nicht zuletzt die gebaute Umgebung, das sozioökonomische und politische Umfeld.

Mit Bewegungsförderung wird letztlich das Ziel verfolgt, die Gesundheit, das Wohlbefinden sowie die Lebensqualität der Bevölkerung zu verbessern. Wobei die Einhaltung der Grundprinzipien der Gesundheitsförderung die Chance erhöht, dass die Investitionen ressourcenschonend und effektiv eingesetzt werden.

Mit dem Nationalen Aktionsplan Bewegung (NAP.b) [39], dem Österreichischen Gesundheitsziel 8 „Gesunde und sichere Bewegung im Alltag durch entsprechende Gestaltung der Lebenswelten fördern“ [56], dem Masterplan Radfahren [57] oder dem Masterplan Gehen [58] verfügt Österreich über mehrere spezifische Leitdokumente, die als Argumentationsgrundlage in der Diskussion um notwendige Investitionen herangezogen werden können. Investitionen, die sich, so zeigen zahlreiche Studien, langfristig rechnen.

\section{Danksagung}

Die Erstellung der Österreichischen Bewegungsempfehlungen und diese open access Publikation wurden dankenswerter Weise von der Gesundheit Österreich $\mathrm{GmbH}$, Geschäftsbereich Fonds Gesundes Österreich, und der Österreichischen Gesellschaft für Public Health unterstützt.

\section{Interessenkonflikt}

Die Autorinnen und Autoren geben an, dass kein Interessenkonflikt besteht.

\section{Literatur}

[1] Felder-Puig R, Teutsch F, Ramelow D et al. Gesundheit und Gesundheitsverhalten von österreichischen Schülerinnen und Schülern. Ergebnisse des WHO-HBSC-Survey 2018. Wien: Bundesministerium für Arbeit, Soziales, Gesundheit und Konsumentenschutz; 2019 
[2] Felder-Puig R, Teutsch F, Ramelow D et al. Gesundheit und Gesundheitsverhalten von österreichischen Lehrlingen. Unveröffentlicht. Wien: Institut für Gesundheit und Prävention; 2019

[3] Fonds Gesundes Österreich. Österreichische Empfehlungen für gesundheitswirksame Bewegung. Wien: Eigenverlag; 2010

[4] Statistik Österreich. Österreichische Gesundheitsbefragung 2014: Hauptergebnisse des Austrian Health Interview Survey (ATHIS) und methodische Dokumentation. Wien; 2015

[5] Finger JD, Tafforeau J, Gisle L et al. Development of the European Health Interview Survey - Physical Activity Questionnaire (EHIS-PAQ) to monitor physical activity in the European Union. Arch Public Health 2015; $73: 59$

[6] Dorner TE, Wilfinger J, Hoffman $\mathrm{K}$ et al. Association between physical activity and the utilization of general practitioners in different age groups. Wien Klin Wochenschr 2019; 131: 278-287

[7] European Union. Special Eurobarometer 472 - Wave EB88.4. Sport and physical activity (2018). Im Internet: ec.europa.eu/sport/ news/2018/new-eurobarometer-sport-and-physical-activity_en, Stand: 22.04 .2020

[8] Bundesministerum für Öffentlichen Dienst und Sport. BewegungsMonitoring. Bevölkerungsbefragung 2017: Kurzbericht. Wien: Bundesministerium für Öffentlichen Dienst und Sport; 2018

[9] Bundesministerum für Öffentlichen Dienst und Sport. BewegungsMonitoring. Bevölkerungsbefragung 2017: Tabellenband. Wien: Bundesministerium für Öffentlichen Dienst und Sport; 2017

[10] Statistik Austria. Österreichische Gesundheitsbefragung 2014: Hauptergebnisse des Austrian Health Interview Survey (ATHIS) und methodische Dokumentation. Wien: Bundesministerium für Gesundheit; 2015

[11] Stein KV, Rieder A, Dorner TE. East-West gradient in cardio-vascular mortality in Austria: how much can we explain by following the pattern of risk factors? Int J Health Geogr 2011; 10: 59

[12] World Health Organization. Growing up unequal: gender and socioeconomic differences in young people's health and well-being: HEALTH BEHAVIOUR IN SCHOOL-AGED CHILDREN (HBSC) STUDY: INTERNATIONAL REPORT FROM THE 2013/2014 SURVEY. Copenhagen: World Health Organization

[13] Allender S, Foster C, Scarboroug P et al. The burden of physical activity-related ill health in the UK. J Epidemiol Community Health 2007; 61: 344-348

[14] Breyer F, Zweifel P, Kifmann M.. Gesundheitsökonomik. Berlin, Heidelberg: Springer-Verlag; 2012

[15] European Commission. EU burden from non-communicable diseases and key risk factors. 2017/m Internet ec.europa.eu/jrc/en/healthknowledge-gateway/societal-impacts/burden Stand: 24.04.2019

[16] Forouzanfar MH, Afshin A, Alexander LT et al. GBD 2015 Risk Factors Collaborators. Global, regional, and national comparative risk assessment of 79 behavioural, environmental and occupational, and metabolic risks or clusters of risks, 1990-2015: a systematic analysis for the Global Burden of Disease Study 2015. Lancet 2016; 388: 1659-1724

[17] World Health Organization. Global Strategy on Diet, Physical Activity and Health. Im Internet: www.who.int/dietphysicalactivity/pa/en/; Stand: 28.04 .2019

[18] Lee IM, Shiroma E], Lobelo F et al. Effect of physical inactivity on major non-communicable diseases worldwide: an analysis of burden of disease and life expectancy. Lancet 2012; 380: 219-229

[19] Abell JE, Hootman JM, Zack MM et al. Physical activity and health related quality of life among people with arthritis. J Epidemiol Community Health 2005; 59: 380-385

[20] Ding D, Lawson KD, Kolbe-Alexander TL et al. The economic burden of physical inactivity: a global analysis of major non-communicable diseases. Lancet 2016; 388: 1311-1324
[21] Drummond MF, Sculpher, Claxton K et al. Methods for the economic evaluation of health care programmes. Oxford: Oxford University Press; 2015

[22] Mattli R, Wieser S, Probst-Hensch N et al. Physical inactivity caused economic burden depends on regional cultural differences. Scand J Med Sci Sports 2019; 29: 95-104

[23] Foster C, Allender S. Costing the burden of ill health related to physical inactivity for Scotland. NHS Health Scotland; 2013

[24] Mayer S, Paulus ATG, Łaszewska A et al. Health-Related Resource-Use Measurement Instruments for Intersectoral Costs and Benefits in the Education and Criminal Justice Sectors. Pharmacoeconomics 2017; 35: 895-908

[25] Idler N, Teuner CM, Hunger M et al. The association between physical activity and healthcare costs in children - results from the GINIplus and LISAplus cohort studies. BMC Public Health 2015; 15: 437

[26] World Health Organization. Chronic diseases and their common risk factors (2016). Im Internet: www.who.int/chp/chronic_disease_report/ media/Factsheet1.pdf; Stand: 22.04.2020

[27] Dorner TE. Public Health Herausforderungen in Bezug auf körperliche Aktivität. Sport-und Präventivmedizin 2009; 39: 37

[28] World Health Organization. 10 key facts on physical activity in the WHO European Region. Im Internet: www.euro.who.int/en/healthtopics/disease-prevention/physical-activity/data-and-statistics/10-keyfacts-on-physical-activity-in-the-who-european-region; Stand: 21.06.2019

[29] Weiß O Sport und Gesundheit. Die Auswirkungen des Sports auf die Gesundheit - eine sozio-ökonomische Analyse. Wien: Bundesministerium für Soziale Sicherheit und Generationen, Bundessportorganisation

[30] Weiß O, Pichlmaier A, Hanisch W et al. Auswirkungen von Sport auf die Gesundheit. Österreichische Ärztezeitung 2016; 9: 20-26

[31] Statistik Austria. Gesundheitsausgaben in Österreich laut System of Health Accounts (SHA) 2004-2017, in Mio. (2019). Im Internet: www. statistik.at/web_de/statistiken/menschen_und_gesellschaft/ gesundheit/gesundheitsausgaben/019701.html; Stand: 22.04.2020

[32] Roux L, Pratt M, Tengs TO et al. Cost effectiveness of communitybased physical activity interventions. Am J Prev Med 2008; 35 : 578-588

[33] Alt R, Binder A, Helmenstein C et al. Der volkswirtschaftliche Nutzen von Bewegung. Volkswirtschaftlicher Nutzen von Bewegung, volkswirtschaftliche Kosten von Inaktivität und Potenziale von mehr Bewegung. Studie im Auftrag der Österreichischen Bundes-Sportorganisation und Fit Sport Austria. Wien: SportsEconAustria Institut für Sportökonomie; 2015

[34] Leoni T, Böheim R. Fehlzeitenreport 2018: Kranksheits- und unfallbedingte Fehlzeiten in Österreich - Präsentismus und Absentismus. Wien: Österreichisches Institut für Wirtschaftsforschung im Auftrag von Bundesarbeitskammer, Wirtschaftskammer Österreich und Hauptverband der österreichischen Sozialversicherungsträger; 2018

[35] Sallis JF, Cervero RB, Ascher W et al. An ecological approach to creating active living communities. Annu Rev Public Health 2006; 27 : 297-322

[36] Heneweer H, Vanhees L, Picavet HS. Physical activity and low back pain: a U-shaped relation? Pain 2009; 143: 21-25

[37] Rütten A, Pfeifer K, Rütten A. Eds. Forschung und Praxis der Gesundheitsförderung: Sonderheft 3. Nationale Empfehlungen für Bewegung und Bewegungsförderung (Aufl. 1.2.06.17). Erlangen-Nürnberg: Bundeszentrale für gesundheitliche Aufklärung (BZgA). 2017

[38] World Health Organization. Global action plan on physical activity 2018-2020: more active people for a healthier world. Geneva: World Health Organization; 2018 
[39] Bundesministerium für Gesundheit und Frauen Ed. Gesundheitsziele Österreich: Richtungsweisende Vorschläge für ein gesünderes Österreich - Langfassung. Wien: Bundesministerium für Gesundheit und Frauen; 2017

[40] Dahlgren G, Whitehead M. Policies and strategies to promote social equity in health. Stockholm: Institute for future studies; 1991

[41] Gollner E, Szabo B, Schnabel F et al. Gesundheitsförderung konkret - Ein forschungsgeleitetes Lehrbuch für die Praxis. Wien: Holzhausen; 2018

[42] Fonds Gesundes Österreich. Aktive Mobilität - Argumentarium KOMPAKT. Intersektorale Argumente zur Förderung Aktiver Mobilität in Österreich. Wien: Fonds Gesundes Österreich; 2018

[43] Bock F de. Ed. Bewegungsförderung im Kindes- und Jugendalter. Düsseldorf: Landesinstitut für Gesundheit und Arbeit des Landes Nordrhein-Westfalen; 2011

[44] Hills AP, Dengel DR, Lubans DR. Supporting public health priorities: recommendations for physical education and physical activity promotion in schools. Prog Cardiovasc Dis 2015; 57: 368-374

[45] Fonds Gesundes Österreich. Aktive Mobilität - gesund unterwegs! Beispiele aus der Praxis für Schule und Kindergarten. Wien: Fonds Gesundes Österreich; 2019

[46] Stoffel S, Gröben F, Pronk NP et al. Bewegungsförderung im Betrieb ein wichtiger Baustein der multifaktoriell konzipierten Betrieblichen Gesundheitsförderung. Düsseldorf: Landesinstitut für Gesundheit und Arbeit des Landes Nordrhein-Westfalen. 2011

[47] Pratt M, Perez LG, Goenka S et al. Can population levels of physical activity be increased? Global evidence and experience. Prog Cardiovasc Dis 2015; 57: 356-367

[48] Fonds Gesundes Österreich. Aktive Mobilität - gesund unterwegs! Beispiele aus der Praxis für Betriebe. Wien: Fonds Gesundes Österreich; 2019

[49] Bucksch J, Claßen T, Schneider S. Eds. Bewegungsförderung auf kommunaler Ebene. Düsseldorf: Landesinstitut für Gesundheit und Arbeit des Landes Nordrhein-Westfalen, Düsseldorf; 2011
[50] Geuter G, Hollederer A. Eds. Bewegungsförderung für ältere und hochaltrige Menschen. Düsseldorf: Landesinstitut für Gesundheit und Arbeit des Landes Nordrhein-Westfalen; 2011

[51] Spicker I, Lang G. Kommunale Gesundheitsförderung mit Fokus auf ältere Menschen. Wien: Fonds Gesundes Österreich; 2011

[52] Ralf C, Krupp S, Willkomm M et al. Das „Lübecker Modell Bewegungswelten“ - Multidimensionale Prävention in stationären Pflegeeinrichtungen durch ein bewegungsförderndes Programm. Bundesgesundheitsblatt, Gesundheitsforschung 2019; 62: 267-273

[53] Mackay G], Neill JT. The effect of "green exercise" on state anxiety and the role of exercise duration, intensity, and greenness: A quasi-experimental study. Psychology of Sport and Exercise 2010; 11: 238-245

[54] Morita E, Imai M, Okawa M et al. A before and after comparison of the effects of forest walking on the sleep of a community-based sample of people with sleep complaints. Biopsychosoc Med 2011; 5: 13

[55] Szabo B. Einflüsse der Natur auf das Bewegungsverhalten junger Erwachsener: Eine quantitative Erhebung zur Bedeutung von Naturgebieten als Bewegungsstätten in den Bundesländern Burgenland und Tirol. Unveröffentlichte Bachelorarbeit. Pinkafeld: FH Burgenland; 2014

[56] Bundesministerium für Gesundheit und Frauen. Ed. Gesundheitsziele Österreich: Richtungsweisende Vorschläge für ein gesünderes Österreich - Langfassung. Wien: Bundesministerium für Gesundheit und Frauen; 2017

[57] Bundesministerium für Land- und Forstwirtschaft, Umwelt und Wasserwirtschaft. Masterplan Radfahren 2015-2025. Wien: Bundesministerium für Land- und Forstwirtschaft, Umwelt und Wasserwirtschaft, Wien; 2015

[58] Bundesministerium für Land- und Forstwirtschaft, Umwelt und Wasserwirtschaft. Masterplan Gehen: Strategien zur Förderung des FußgängerInnenverkehrs in Österreich. Wien: Bundesministerium für Land- und Forstwirtschaft, Umwelt und Wasserwirtschaft; 2015

[59] Fonds Gesundes Österreich nach Dahlgren G, Whitehead M. 1991; Im Internet: fgoe.org/medien/grafiken; Stand: 22.04.2020 